\title{
Alter, Altersversorgung und Altersmedizin in der Geschichte*
}

Dorothee Guggenheimer

\footnotetext{
Der vorliegende Text basiert auf den Beiträgen von Dorothee Guggenheimer und Stefan Sonderegger im von Dr. med. Christoph Hürny, PD Dr. Stefan Sonderegger und lic. phil. Dorothee Guggenheimer herausgegebenen Buch «Achtung Alter! Zum 25jährigen Bestehen der Geriatrischen Klinik und des Pflegeheims am Bürgerspital St. Gallen. St. Gallen; 2005.
}

Korrespondenz:

lic. phil. Dorothee Guggenheimer Stadtarchiv der

Ortsbürgergemeinde St. Gallen

Notkerstrasse 22

CH-9000 St. Gallen

dorothee.guggenheimer@

ortsbuerger.ch
Die Generation der Senioren drang in den letzten 25 Jahren immer mehr ins öffentliche Bewusstsein: So besuchen z.B. Pensionierte die Universität, Siebzigjährige laufen Marathon, Achtzigjährige benützen Mobiltelefone und schreiben E-Mails. Mit Mulmigkeit wird zudem konstatiert, dass vor 400 Jahren etwa 1,5 Prozent und noch vor 100 Jahren lediglich 5 Prozent der mitteleuropäischen Bevölkerung über 65 Jahre alt waren, während es heute bald 20 Prozent der Bevölkerung sein werden [1].

Die geschilderten Entwicklungen haben die Diskussion über das Alter in den letzten Jahren und Jahrzehnten verstärkt. Viele verherrlichen dabei die Vergangenheit: Sie zitieren Ciceros Lobreden des Alters und betonen, früher sei die ältere Generation im trauten Kreise der eigenen Kinder und Kindeskinder aufgehoben gewesen, während Alte heute oft lieblos in Alters- und Pflegeheime abgeschoben würden. Verbrachten in einer Zeit ohne Sozialversicherung und ohne Pensionsberechtigung «durchschnittliche» Betagte ihren Lebensabend wirklich im Kreise ihrer Familie? Und wie gestaltete sich die Altersmedizin und -pflege in den früheren Jahrhunderten? Diese Fragen werden im vorliegenden Beitrag behandelt.

\section{Alter in der Geschichte}

Unser Lebenslauf wird gemeinhin in klar definierte Lebensphasen wie z.B. Kindheit, Ausbildung, Erwerbsarbeit und Ruhestand eingeteilt. Daraus zu schliessen, dass bereits im Mittelalter und in der Frühen Neuzeit eine ebensolche Gleichförmigkeit der Lebensalter geherrscht habe, wäre jedoch nicht korrekt. Dass Individuen ein hohes Alter erreichen konnten, war zwar nicht ausgeschlossen, oft verlief das Leben jedoch anders: Aufgrund von Seuchen, Hungersnöten und Kriegen war der Tod in jedem Lebensalter präsent, und der Beginn einer eigentlichen Altersphase konnte - sofern überhaupt ein höheres Alter erreicht wurde - nicht einheitlich festgelegt werden. Die konkrete Ausgestaltung des Alters war sehr unterschiedlich. Sie war u. a. abhängig von der sozialen und wirtschaftlichen Lage der älteren Menschen. Zudem unterschied sich die Situation älterer Menschen auf dem Land und in der Stadt.

\section{Alter auf dem Land}

Über die Lebenserwartung der mittelalterlichen und frühneuzeitlichen bäuerlichen Gesellschaft gibt es zahlreiche Untersuchungen. Eine jüngst vorgenommene medizin-anthropologische Untersuchung von Gräbern des 16. und 17. Jahrhunderts aus der Gemeinde Lütisburg (Toggenburg) liefert Hinweise auf das Alter der bäuerlichen Bevölkerung [2]. Insgesamt konnten Skelettreste 54 Menschen zugeordnet werden. Der demographische Befund ergab, dass 43 Prozent dieser Menschen im juvenilen Alter starben. Bei den untersuchten Skeletten überwiegen Kleinkinder, Kinder und Jugendliche, während von über 60jährigen überhaupt keine Skelette gefunden wurden. Der zuständige Anthropologe Bruno Kaufmann sieht den Grund für dieses junge Todesalter in der Unter- und Fehlernährung sowie der harten körperlichen Arbeit, die das Leben der Menschen geprägt haben.

Die eigene Landwirtschaft war die Existenzgrundlage für die meisten Menschen. Die ganze Familie war in den Arbeitsprozess eingespannt. Der Verlust des Ehepartners wog nicht nur persönlich, sondern auch wirtschaftlich schwer und musste - wenn möglich - durch eine Wiederverheiratung oder durch eine Haushaltszusammenlegung wettgemacht werden. Starb der Ehemann und Vater einer Bauernfamilie, konnte es auch zu einer vorzeitigen Hofübergabe an Nachkommen kommen. In der älteren Geschichtsschreibung wird zudem die Vorstellung von grossen, drei bis vier Generationen umfassenden Haushalten auf dem Land vertreten. Vor dem 19. Jahrhundert lebten aber nachweislich nur selten drei oder gar vier Generationen unter einem Dach zusammen [3]. Dies hat verschiedene Gründe (u.a. mangelnder Platz für eine grosse Anzahl Personen) und ist schon angesichts der viel geringeren Lebenserwartung einleuchtend. Von vornherein ausgeschlossen von dieser Möglichkeit blieben die Menschen, die ihr Leben lang als Dienstboten gearbeitet hatten und nie verheiratet sowie kinderlos waren. Konnten sie nicht mehr arbeiten, so stand zu ihrer Versorgung meist nur die «Einlege» zur Verfügung: Sie wurden reihum von Hof zu Hof weitergereicht und erhielten von einem Bauern nach dem andern 


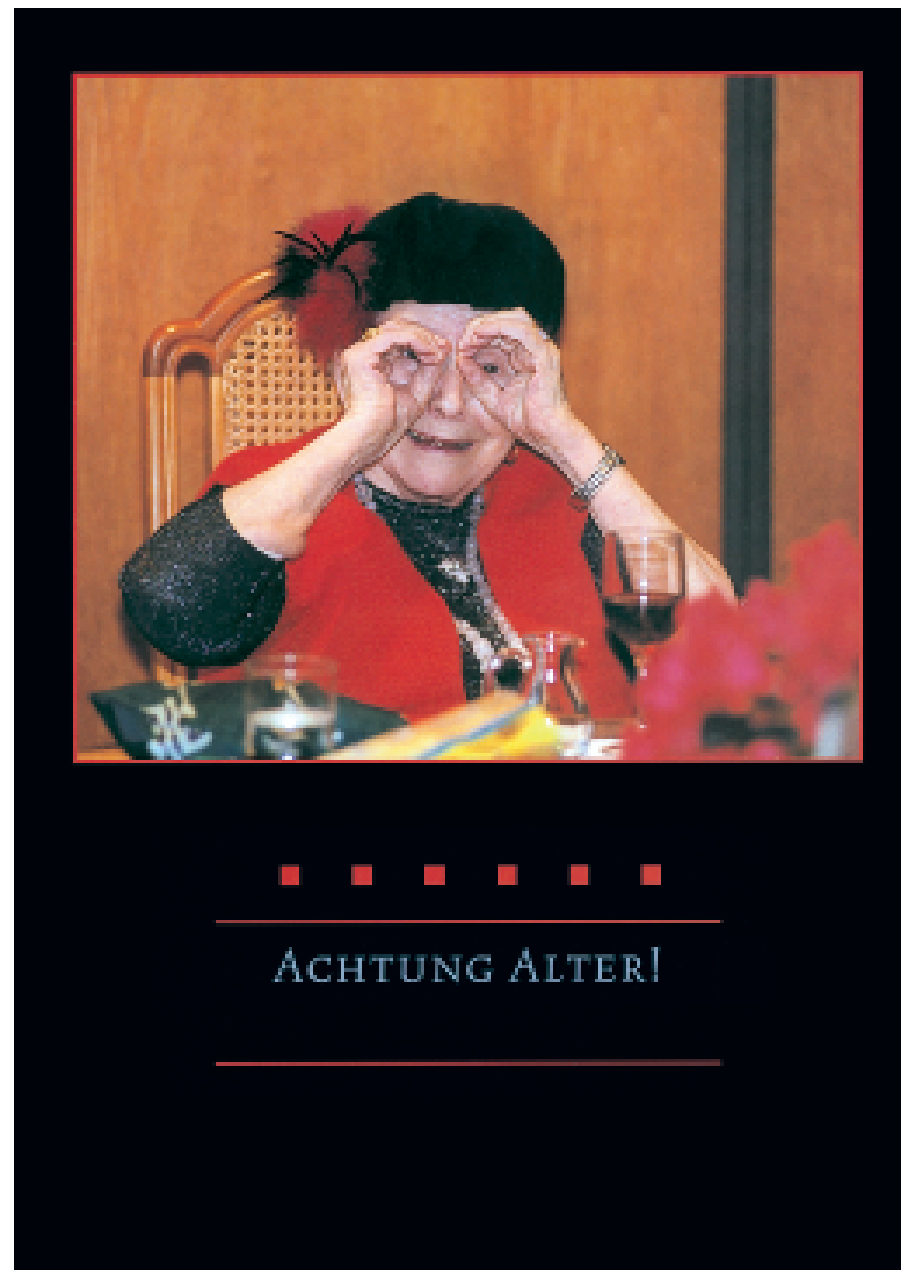

kurzfristig Kost und Logis [3]. Die überlieferten Dokumente beschreiben ein sehr demütigendes «Geduldetsein», erwähnen einen Schlafplatz in der Ecke des Stalls und einen Essplatz weitab von den andern Familienmitgliedern [3].

\section{Alter in der Stadt}

Auch bei den Stadtbewohnerinnen und -bewohnern lässt sich keine bestimmte Phase ausmachen, die als «Alter» zu bezeichnen war. Beginn und Verlauf der Altersphase hingen unter anderem ab vom sozialen und rechtlichen Status der Stadtbewohner. Vermögende Bürger beispielsweise waren von relativ wenigen sozialen Risiken betroffen. Sie konnten im Falle einer altersbedingten Kräftereduktion oft von Zinsen leben. Ihr Besitz erlaubte ihnen zudem, sich gegebenenfalls im Spital, das damals auch die Funktion eines Altersheims innehatte, gegen einen hohen Betrag einzuquartieren.

Anders gestaltete sich das Leben von Handwerkern, deren Einkommen nicht auf Besitz und Vermögen, sondern auf steter eigener Arbeit beruhte. Für sie bestand in der nachlassenden körperlichen Kraft ein grosses Risiko, denn nur selten waren sie in der Lage, sich für ihr Alter Ersparnisse auf die Seite zu legen. Dieser Schwierigkeit war man sich schon im Mittelalter bewusst. Ältere Menschen konnten - oft jedoch nur, wenn sie das Bürgerrecht der jeweiligen Stadt besassen bei der Obrigkeit um meist bescheidene Unterstützung bitten. Dazu war in manchen Städten eine Reihe von Berufen - z.B. Torwächter oder Rathausdiener - der älteren Generation vorbehalten, die nicht mehr ihren ursprünglichen Beruf ausüben konnte.

Wer so schwach war, dass er gar keiner Tätigkeit mehr nachgehen konnte, der musste seinen Unterhalt durch Bettel bestreiten, denn die Versorgung von Eltern in den Haushalten ihrer Kinder kam in Städten noch seltener als auf dem Land vor [4].

\section{Alter im Spital}

Eine Alternative für besonders zahlungskräftige Alte oder aber ganz arme und nicht mehr arbeitsfähige Alte war das Spital. In ein Spital konnte man sich gegen einen hohen Geldbetrag einkaufen, oder man wurde «um gotz willen» - um Gottes willen, also ohne Bezahlung - aufgenommen. Voraussetzung für eine unentgeltliche Aufnahme, z.B. in das St.Galler Heiliggeist-Spital, war, dass man bereits zu schwach war, um noch betteln zu können. So bestand für das Spital die Gewissheit, dass es nur Alte unterstützte, die sich wirklich nicht mehr selbst helfen konnten. Zudem konnte das Spital sicher sein, dass diese Alten nicht mehr während allzu langer Zeit auf Kosten des Spitals leben würden. Wer noch dazu in der Lage war, wurde für Arbeiten im Betrieb eingesetzt, zum Flicken von Kleidern oder Schuhen, zum Holzspalten, Bodenwischen usw.

Nebst den armen Spitalbewohnern, die «Siechen-» oder «Muespfründner» genannt wurden, wurden auch Waisen und gebärende Frauen sowie wohlhabende Personen aufgenommen. Letztere mussten entsprechend viel dafür bezahlen, genossen aber einen hohen Lebensstandard. Während die armen Siechenpfründner vor allem Brei und Suppen zu essen bekamen, erhielten die Herrenpfründner, wie die oberste Pfrundkategorie in St. Gallen genannt wurde, Fleisch, Fisch sowie ausreichend Wein (bis zu zwei Liter pro Person und Tag). Die Aufnahme von reichen Alten lohnte sich: Durch sie konnte das Spital den eigenen Besitz im Laufe der Zeit wesentlich erweitern, denn diese Personen vermachten bzw. mussten dem Spital Güter auf dem Land oder Liegenschaften in der Stadt vermachen. So entwickelten sich mittelalterliche und frühneuzeitliche Spitäler oft zu eigentlichen Wirtschafts- 
betrieben mit sozialem Auftrag. Pflege hingegen war - anders als heute - keine hauptsächlich im Spital ausgeübte Tätigkeit (vgl. die entsprechenden Stellen bei [5]).

\section{Alterspflege in der Geschichte}

Obschon ältere Menschen innerhalb von Medizin und Pflege seit Jahrhunderten eine der wichtigsten Zielgruppen darstellen, kam ihnen bis ins 20. Jahrhundert kein besonderer medizinischer Wissenschaftsbereich und damit auch keine spezielle Pflegeform zu. Der wissenschaftliche Begriff «Geriatrie» wurde erst von 1914 an geprägt. Der Begriff «Geriatrie» gewann mit der Publikation des in Wien geborenen Arztes I. L. Nascher zunehmende Bedeutung [6].

\section{Die Entwicklung des Hospitalwesens}

Im Früh- und Hochmittelalter wurden pflegerische Aufgaben in erster Linie von Klöstern wahrgenommen, doch im Laufe des 12. Jahrhunderts kämpfte die Kirche zunehmend gegen die ausgeprägte Pflegetätigkeit von Mönchen und Nonnen an. Oft übernahmen Familienmitglieder pflegerische Aufgaben, sogar Operationen fanden häufig unter Assistenz von Familienmitgliedern in den Wohnhäusern der Kranken statt [7]. Da die Krankenpflege damals nicht im Zentrum der Aufgaben der Spitäler stand, wurden Spitäler je länger, je mehr zu sogenannten Pfrundhäusern. Nur in grösseren Spitälern übernahm so genanntes «Wartpersonal» die eigentliche Pflege, meist waren dies jedoch Knechte oder Mägde ohne jegliche Vorbildung. mien könnte eine Folge verminderter Virulenz von Bakterien und Viren und/oder eine Folge von verbesserten Abwehrkräften der Menschen sein. Vertreter dieser These ist u.a. [13].

b Die neu aufkommende Heimarbeit garantierte eine gewisse Einkommenssicherheit. Die in der Heimarbeit Tätigen konnten deshalb früher heiraten als in den letzten Jahrhunderten, wo der Familiengründung zur finanziellen Versorgung z. B. die Übernahme eines Hofes vorausgehen musste, was je nach Lebensdauer der Eltern lange auf sich warten liess; auch konnte, wenn mehrer Erben vorhanden waren, das Erbe so zerstückelt werden, dass durch den kleinen Erbteil kein sicheres Auskommen garantiert war. Die Heimarbeit hingegen sicherte schon früh das Auskommen und ermöglichte deshalb frühere Eheschliessungen. Daher konnten Frauen durch frühe Heirat und somit den frühen Beginn ihrer Geburtenphase mehr Kinder auf die Welt bringen, was die Bevölkerung ansteigen liess. Vertreter dieser These sind [15].
Bezeichnung als sehr gewichtig einzuschätzen. Ein Grund dafür war, dass studierte Ärzte wohl kaum die ganze Bevölkerung hätten versorgen können, denn es gab zu wenige, um Bewohnern von Kleinstädten oder der Landbevölkerung ausreichende medizinische Versorgung zukommen zu lassen [9].

Frühere pflegerische Aspekte des St.Galler Heiliggeist-Spitals, des Vorgängers des heutigen Bürgerspitals mit Altersheim, Pflegeheim und Geriatrischer Klinik, lassen sich aus dem St. Galler Satzungsbuch von 1561 nachzeichnen; in anderen Teile Europas hatten Pflegende wohl ähnliche Aufgaben. Die Pfleger mussten «den Krankhen pflegen und zusehen, es seye mit Aufwarten, Lupfen, Tragen, Wachen, Saüberen, Wäschen und anderen Nothwendigkeiten. Die Schwachen mit Brühen, Kochen und anderem erquikhen, freündtlich zusprechen, beten und in anderen christlichen Übungen ihr bestes thun. [...] Die Gemach oder Stuben samt den Krankhnen sauber halten, damit kein Unziefer bei ihnen zu oder überhand nemme, sonder mit täglichen Säuberen vertreiben [...]» [10]. Die pflegerischen Leistungen waren in der Frühen Neuzeit also sehr umfassend. Die Wärterinnen und Wärter pflegten, kochten, putzten und beteten mit den Kranken. Daran ist erkennbar, dass die Krankenpflege auch nach der Reformation von religiösen Motivationen geprägt war.

\section{Die «Krise» der Krankenversorgung im 18. Jahrhundert?}

Ältere Darstellungen der Pflege und Medizin haben das 18. Jahrhundert jeweils als «dunkle» Epoche geschildert. Diese Wertung mag damit zusammenhängen, dass die Spitäler im 18. Jahrhundert hoffnungslos überfüllt waren. Dies wiederum hing mit einer Bevölkerungszunahme im 18. Jahrhundert zusammen (vgl. den allgemeinen Überblick bei [11]). Die Gründe für diesen Bevölkerungsanstieg liegen in einem signifikanten Rückgang der Sterblichkeit [12]: Einerseits, weil keine schweren Epidemien mehr - wie z.B. die Pest - grosse Bevölkerungsteile hinwegrafften $^{\mathrm{a}}$, andererseits, weil die allgemeine und die Kindersterblichkeit zurückgingen, da in verschiedenen Gebieten agrarische Innovationen den Übergang zur biologisch vollwertigeren Milch-Kartoffel-Nahrung einleiteten [14]. Zudem war im 18. Jahrhundert auch eine steigende Fertilität ausschlaggebend für einen Bevölkerungsanstieg. ${ }^{\mathrm{b}}$

Der nach wie vor übliche, aber veraltete Aufnahmemodus der Spitäler hatte unter diesen Umständen fatale Konsequenzen: Kranke, Schwangere, Alte, Bettler, Vagabunden, Waisen sowie 
Menschen, die nach heutigem Verständnis eher in eine psychiatrische Klinik als in ein Spital gehörten, drängten sich auf engstem Raum. An genügend ausgebildete Ärzte und Pflegepersonen war nicht zu denken [9].

\section{Die Etablierung der Geriatrie}

Die grossen Fortschritte der naturwissenschaftlichen Medizin (u. a. die Entdeckung der Röntgenstrahlen und des Röntgenapparats, des Penicillins, der Schutzimpfungen sowie der Chemotherapie) wirkten sich am Anfang des 20. Jahrhunderts auf die Aufgaben von Pflegenden und Ärzten aus [9].

Geriatrie ist, wie bereits erwähnt, eine dieser Spezialisierungen, die sich im 20. Jahrhundert etablierte. Die medizinischen Entwicklungen, die zu einer wesentlichen Verlängerung des Lebens führten, machten die Geriatrie erst nötig und möglich. Während gegen Ende des 19. Jahrhunderts die durchschnittliche Lebenserwartung eines Neugeborenen noch 37 Jahre betragen hatte, wurden Menschen im Jahre 1980 rund 70 Jahre alt. Um 1900 machte der Anteil der akuten Krankheiten an der Gesamtsterblichkeit etwa 85 Prozent aus, und 1980 betrug er noch 10 Prozent. Die gewonnenen Lebensjahre brachten eine Zunahme der chronischen Krankheiten mit sich wie z.B. degenerative Veränderungen des Bewegungssystems, Herz-Kreislauf-Erkrankungen, zerebrale Ausfallserscheinungen, chronische Krankheitsprozesse der Nieren, der Leber, des Darmtraktes usw. [9].

Der Blick auf die Entwicklung der Altersversorgung über die Jahrhunderte hinweg legt grosse Unterschiede zwischen früher und heute, aber auch erstaunliche Ähnlichkeiten offen: So hat zum Beispiel eine staatliche finanzielle Unterstützung wie die heutige AHV noch keine lange Tradition; mit dem Spital aus dem 13. Jahrhundert bestand jedoch schon im Mittelalter eine soziale Einrichtung in der Stadt, die auch Mittellose aufnahm. Ebenso fällt auf, dass sowohl früher wie heute die wirtschaftlich Schwachen am stärksten unter den Folgen des Alters leiden.

\section{Literatur}

1 Borscheid P. Geschichte des Alters. Vom Spätmittelalter bis zum 18. Jahrhundert. München: dtv; 1989.

2 Der Ausgrabungsbericht ist bei der Kantonsarchäologie St. Gallen einzusehen.

3 Ehmer J. Sozialgeschichte des Alters. Frankfurt: Suhrkamp; 1990.

4 Ehmer J. Zur Stellung alter Menschen in Haushalt und Familie. In: Conrad C, von Kondratowitz H-J (Hrsg.). Gerontologie und Sozialgeschichte. Berlin: Deutsches Zentrum für Altersfragen; 1983. S. 187-216.

5 Sonderegger S. Landwirtschaftliche Entwicklung in der spätmittelalterlichen Nordostschweiz. St. Gallen: Staatsarchiv; 1994.

6 Nascher IL. Geriatrics. New York; 1914.

7 Sticker A. Die Entstehung der neuzeitlichen Krankenpflege. Stuttgart: Kohlhammer; 1960.

8 Schnipperges H. Die Kranken im Mittelalter. München: C. H. Beck; 1990.

9 Seidler E, Leven KH. Geschichte der Medizin und der Krankenpflege. 7., überarbeitete und erweiterte Auflage. Stuttgart: Kohlhammer; 2003.

10 Ziegler E. Das Heiliggeist-Spital. Ordnungen und Satzungen. In: Ad infirmorum custodium. Zur Einweihung der Geriatrischen Klinik. 750 Jahre Heiliggeist- und Bürgerspital in St. Gallen. St. Gallen; 1980. S. 19-21.

11 Pfister C. Bevölkerung, Klima und Agrarmodernisierung 1525-1860. Bd. II: Das Klima der Schweiz von 1525-1860 und seine Bedeutung in der Geschichte von Bevölkerung und Landwirtschaft. Bern, Stuttgart: Haupt; 1984.

12 Imhof AE. Die gewonnenen Jahre. Von der Zunahme unserer Lebensspanne seit dreihundert Jahren oder von der Notwendigkeit einer neuen Einstellung zu Leben und Sterben. München: C. H. Beck; 1981.

13 Lee WR. Population Growth, Economic Development and Social Change in Europe, 1750-1970. In: Lee WR (ed.). European Demography and Economic Growth. London: Croom Helm; 1979. p. 10-26.

14 Mattmüller M. Das Einsetzen der Bevölkerungswelle in der Schweiz. Versuch eines Überblicks über den Stand der Forschung. Vierteljahresschrift für Sozial- und Wirtschaftsgeschichte 1976;63/3:390-405.

15 Kriedte P, Medick H, Schlumbohm J. Industrialisierung vor der Industrialisierung. Gewerbliche Warenproduktion auf dem Lande in der Formationsperiode des Kapitalismus. Göttingen: Vandenhoeck \& Ruprecht; 1978. 